Neurosurg Focus 17 (5):E6, 2004

\title{
Unruptured intracranial aneurysms: natural history and management decisions
}

\author{
Leodante B. Da Costa, M.D., Thorsteinn Gunnarsson, M.D., M.Sc., \\ and M. Christopher Wallace, M.D., M.Sc. \\ Division of Neurosurgery, Toronto Western Hospital, University of Toronto, Canada; \\ and Division of Neurosurgery, Fundacao Benjamin Guimares/Hospital da Baleia, \\ Belo Horizonte, Minas Gerais, Brazil
}

\begin{abstract}
Aneurysmal subarachnoid hemorrhage (SAH) carries a grim prognosis, with high mortality and morbidity rates. The mortality rate in the first 30 days postrupture is estimated to be in the range of 40 to $50 \%$, and almost half of the survivors will be left with a neurological deficit. Unlike patients with aneurysmal SAH, those with unruptured intracranial aneurysms usually experience no neurological deficit, and their treatment is prophylactic, aiming to reduce the risk of future bleeding and its consequences. The risk of rupture therefore assumes special importance when making decisions regarding which patient or aneurysm to treat.

In previous reports the risk of bleeding for unruptured aneurysms has been stated as approximately $2 \%$ per year. The retrospective part of the International Study of Unruptured Intracranial Aneurysms (ISUIA) reported very low annual bleeding rates $(0.05-1 \%)$ and high surgical morbidity and mortality rates $(8-18 \%)$, prompting discussion in which the benefits of prophylactic treatment in the majority of these lesions were questioned. Prospective data from the second part of the ISUIA recently included rupture rates ranging from 0 to $10 \%$ per year. The aim of this paper was to review the evidence that is currently available for neurosurgeons to use when making decisions regarding patients who would benefit from treatment of an unruptured intracranial aneurysm.
\end{abstract}

\section{KEY WORDS • intracranial aneurysm • unruptured asymptomatic aneurysm • surgical risk}

\section{OVERVIEW}

Aneurysmal SAH carries a grim prognosis, with high mortality and morbidity rates. The estimated mortality rate in the first 30 days post-SAH is in the range of 40 to $50 \%$, and almost half of the survivors will be left with a neurological deficit. Due to the severe impact the SAH has on the brain because of its primary and secondary injury, the outcome has only modestly improved despite great advances in diagnostic imaging, surgical and endovascular treatment, and neurointensive care. Unruptured intracranial aneurysms are usually diagnosed during the investigation of another ruptured lesion. It is also well known that some diseases, such as polycystic kidney disease, Ehler-Danlos and Marfan syndrome, and familial predisposition increase the incidence of these lesions, and patients with these conditions are usually screened early in life for intracranial aneurysms. The easy access to diagnostic imaging, including CT angiography and magnetic resonance angiography, has increased the number of asymptomatic lesions discovered incidentally during investigations of unrelated complaints. 1,2,4,6,7,10,12,21,22,25

Abbreviations used in this paper: $\mathrm{CT}=$ computerized tomography; ISUIA = International Study of Unruptured Intracranial Aneurysms; $\mathrm{MCA}=$ middle cerebral artery; $\mathrm{PCoA}=$ posterior communicating artery; $\mathrm{SAH}=$ subarachnoid hemorrhage.
Because of the catastrophic effects of aneurysmal SAH, when unruptured intracranial aneurysms were discovered, for decades they were excluded from the circulation with surgical clip occlusion and more recently by endovascular therapy. The purpose of this treatment is to prevent future neurological damage or death due to possible aneurysmal bleeding. The practice of repairing unruptured intracranial aneurysms has been continued without strong supporting evidence that it provides real benefits in terms of improving longevity and quality of life.

The most important factor in the management of unruptured intracranial aneurysms is the risk of rupture balanced against the risks associated with treatment. The risks of SAH from an unruptured aneurysm were reported and had been accepted for the past three decades as approximately $2 \%$ per year. With the publication of the first part of the ISUIA in 1998, the natural history figures included an exceedingly low rupture rate $(0.05-1 \%$ per year). With very low rates of hemorrhage, the decision about which patients would benefit became more challenging. New data from the second part of the ISUIA showed rupture rates in the range of $0.5-3.0 \%$ per annum, and stratified the risk according to the lesions' location and size. One clearly appreciates that the natural history of unruptured aneurysms has been a moving target, and that more information is required to understand fully the behavior of these lesions. ${ }^{6,7,14-17,19}$ 
The purpose of this article was to review our current understanding of the natural history of unruptured intracranial aneurysms in light of the new data provided by the prospective arm of the ISUIA,${ }^{6}$ and to match this information with the risks associated with surgical therapy to illustrate how that information is used to influence our management decisions.

\section{Review of Unruptured Intracranial Aneurysm Natural History and Current Guidelines}

Several studies have been published in which the natural history and the risks of available treatments for unruptured aneurysms have been analyzed. Most of these studies are case series from single institutions, some of them with a significant number of patients enrolled, but still with a small number of patients if compared with the recently published ISUIA (Table 1) ${ }^{6,7,11,22,25}$ In the year 2000, the American Heart Association published guidelines for the management of unruptured intracranial aneurysms based on a literature review ${ }^{1}$ that included the retrospective part of the ISUIA. ${ }^{7}$ It is important to note the statement by the American Heart Association that "the recommendations may not apply to all situations. Further anticipated epidemiological research during the next few years, as well as possible subsequent randomized trials for appropriate subgroups of patients with UIA [unruptured intracranial aneurysms], will be useful for confirmation or modification of the guidelines."

Based on the analysis of the available data on unruptured aneurysms, the following recommendations were made. 1) Asymptomatic intracavernous aneurysms should not be treated. In large symptomatic ones the treatment should be individualized. 2) All symptomatic intradural aneurysms should be treated. 3) Incidental aneurysms with diameters less than $10 \mathrm{~mm}$ should not be treated. Nevertheless, lesions approaching $10 \mathrm{~mm}$, those with daughter aneurysm formation, those in young patients or in individuals with a family history of SAH deserve special consideration for treatment. 4) Aneurysms found in association with a ruptured lesion and those with a diameter of more than $10 \mathrm{~mm}$ deserve strong consideration for treatment, especially in young patients.

The consensus committee concluded that there was insufficient evidence in the literature to support standards of care for the treatment of unruptured intracranial aneurysms. At best, the literature contained only Grade IV and $\mathrm{V}^{5}$ evidence, supporting Grade $\mathrm{C}$ recommendations (various potential clinical actions, any of which could be considered appropriate). Three years after the publication of

TABLE 1

Review of the ISUIA and other literature on unruptured aneurysms showing the annual bleeding rate

\begin{tabular}{lcl}
\hline \multicolumn{1}{c}{ Authors \& Year } & No. of Patients & Annual Bleeding Rate (\%) \\
\hline Wiebers, et al., 1987 & 130 & 1.4 \\
Yasui, et al., 1997 & 234 & 2.3 \\
anonymous (ISUIA), 1998 & 2621 & $0.05-1$ \\
Juvela, et al., 2000 & 142 & 1.3 \\
Wiebers, et al., (ISUIA) 2003 & 4060 & $0-10^{*}$ \\
\hline
\end{tabular}

* Based on size and location. See Table 2 for more details. the guidelines, a large, prospective, multicenter study on the natural history of unruptured intracranial aneurysms was published; this was the second part of the ISUIA. This study further improved our understanding of the natural history of hemorrhage in unruptured aneurysms, but has also provided new information that impacts on previous guideline recommendations.

\section{The ISUIA}

Part 1. In the ISUIA, 4060 patients were assessed to determine the outcome of unruptured intracranial aneurysms, whether they underwent surgery, endovascular treatment, or no treatment (observation). In the first part of the study, published in $1998,{ }^{7}$ the risks of rupture and of surgical treatment were evaluated in 2621 patients seen in 53 centers in Canada, the US, and Europe, and the study contained a prospective and a retrospective arm. Retrospectively, the investigators analyzed the natural history of unruptured intracranial aneurysms in 1449 patients with 1937 unruptured lesions. These patients were divided into two groups: one included individuals who had a history of SAH from another aneurysm (722 patients) and the other consisted of individuals with no history of SAH (727 patients). Prospectively, the morbidity and mortality rates related to surgical treatment were determined in 1172 patients in whom unruptured intracranial aneurysms were diagnosed. The investigators did not differentiate between symptomatic and asymptomatic unruptured aneurysms for the enrollment of patients, and excluded lesions with diameters less than $2 \mathrm{~mm}$ as well as mycotic, fusiform, and traumatic aneurysms. The end points of the study were SAH, or intracerebral hemorrhage, or death. The morbidity of the surgical treatment was defined as a Rankin Scale score of 3 to 5 , a score of less than 24 on the Mini-Mental State Examination, or a score less than 27 on the Telephone Interview for Cognitive Status at 30 days and 1 year. The mean follow-up duration was 8.3 years, corresponding to approximately 12,023 patient-years.

The investigators found that the size of the aneurysm, its location, and the history of previous SAH were important in defining the probability of future rupture, which was found to be very low. For patients with no history of SAH whose aneurysms were less than $10 \mathrm{~mm}$ in diameter, the risk of rupture was $0.05 \%$ per year, a rate far lower than had been previously assumed. Aneurysms of the same size in patients with a history of SAH had $0.5 \%$ chance of bleeding per year, almost 11 times higher (but still lower than a rate of $2 \%$ per year). For aneurysms more than $10 \mathrm{~mm}$ in diameter, the annual rupture rates were the same in both groups; approximately $1 \%$. These were considerably lower than previously reported rupture rates ranging from $1.3 \%$ to $2.6 \%$ per year. Giant aneurysms in patients with no history of SAH had a higher rupture rate in the 1st year after diagnosis $(6 \%)$. Larger aneurysms, and those located in the PCoA, vertebral and posterior cerebral arteries, or basilar tip were found to be more prone to rupture in patients with no history of SAH, but in those with such a history, only the basilar tip location was associated with higher rupture rates.

The morbidity and mortality rates associated with surgical treatment that are detailed in the first part of the 
study were also higher than previously reported, mostly because of higher rates of mental status impairment at the 30-day and 1-year follow-up review, which was not evaluated in most of the previous series. The morbidity from endovascular treatment could not be evaluated in the first part of the study because of the small number of patients included. Age was the only significant independent predictor of poor surgical outcome. Location and size of the lesion were other potential predictors of surgical morbidity.

Part 2. In the second part of the study, published in 2003, 4060 patients were prospectively analyzed and outcome for patients undergoing endovascular treatment was also considered. ${ }^{6}$ The untreated group consisted of 1692 patients with 2686 aneurysms, 1077 with no history of SAH and 615 with a history of rupture from another aneurysm. The rules for enrollment and exclusion were the same as in the first part of the ISUIA, mentioned earlier. Of these individuals, only 51 had a confirmed SAH (3\%), most within the first 5 years. Larger aneurysmal size was associated with higher rupture rates in patients with a history of SAH, but not in those without such a history. Location was important in predicting higher rupture rates (Table 2).

For the treated group, which consisted of 1917 patients treated surgically and 451 treated with endovascular coil placement, the mean follow-up period was 4 years. In the surgically treated group, among the variables assessed as potentially predictive of poor outcome, age was the strongest. That is, in patients younger than 40 years and in those 40 to 49 years of age, the rate for poor outcome was 5 to $9 \%$, whereas in those 50 to 59 years old the rate was 10 to $15 \%$, in those 60 to 69 years of age it was 15 to $20 \%$, and in those 70 years of age or older it was 22 to $40 \%$.

Other variants with predictive power were lesion diameter greater than $12 \mathrm{~mm}$, posterior circulation, previous ischemic cerebrovascular disease, and symptomatic unruptured aneurysm. Only location in the posterior fossa and diameter greater than $12 \mathrm{~mm}$ were associated with a

\section{TABLE 2}

Relationship between size and location of aneurysms and the annual and cumulative risk of rupture after 5 years*

\begin{tabular}{llllll}
\hline \hline & \multicolumn{5}{c}{ Size (mm), \% Ruptured $\dagger$} \\
\cline { 2 - 3 } \multicolumn{1}{c}{ Location } & \multicolumn{2}{c}{$<$} & & & \\
\cline { 2 - 5 } & Group 1* & Group 2* & $7-12$ & $13-24$ & $\geq 25$ \\
\hline 5-year risk & & & & & \\
ICA, ACoA/ACA, MCA & 0 & 1.5 & 2.6 & 14.5 & 40 \\
PCoA/post circ & 2.5 & 3.4 & 14.5 & 18.4 & 50 \\
intracavernous ICA & 0 & 0 & 0 & 3.0 & 6.4 \\
annual risk & & & & & \\
ICA, ACoA/ACA, MCA & 0 & 0.3 & 0.52 & 2.9 & 8 \\
PCoA/post circ & 0.5 & 0.68 & 2.9 & 3.68 & 10 \\
intracavernous ICA & 0 & 0 & 0 & 0.6 & 1.28 \\
\hline
\end{tabular}

* Group 1, no history of SAH; group 2, previous SAH from another aneurysm. Abbreviations: $\mathrm{ACA}=$ anterior cerebral artery; $\mathrm{ACoA}=$ anterior communicating artery; ICA $=$ internal carotid artery; post circ $=$ posterior circulation.

$\dagger$ The data were not provided in the original articles because for lesions larger than $7 \mathrm{~mm}$ the presence of previous SAH made no difference in the bleeding rate. worse outcome after endovascular treatment. The authors proposed that for patients with unruptured intracranial aneurysms less than $7 \mathrm{~mm}$ in diameter there was no benefit in treatment, either surgical or endovascular, because of the very low rupture rates $(0.1 \% /$ year) and the morbidity and risk of death related to treatment. Perhaps symptomatic unruptured small aneurysms (which are very rare) could be considered an exception. For patients with no history of SAH whose aneurysms are larger than $7 \mathrm{~mm}$ in diameter, and for all patients with a history of previous SAH from another unruptured intracranial aneurysm, the cumulative rates of bleeding are higher, and are very similar to the rates shown previously in other single-institution series, if we consider size and location. The risks associated with surgical and endovascular treatment were still higher than previously reported, even though in this second part of the trial the surgical risks improved from $15.7 \%$ in Part 1 to $12.6 \%$ in Part 2 in patients with no history of SAH from another lesion, and from $13.1 \%$ in Part 1 to $10.1 \%$ in Part 2 in patients with SAH from another lesion. Age was a strong predictor of poor surgical outcome, and therefore the investigators suggest that treatment options should be analyzed individually, considering the age of the patient and the location and size of the aneurysm.

Endovascular treatment is a very attractive option, both for patients and physicians, and its main drawback is that almost half of the lesions could not be completely occluded with endovascular treatment. Because it is not known whether incomplete coil occlusion of an unruptured aneurysm can change its natural history, the treatment decision needs to be made between a more appealing initial option with an unknown long-term prognosis (endovascular treatment) and a more invasive procedure with a known long-term outcome (surgery). Endovascular treatment could be more suitable for older patients, because age wasn't associated with higher morbidity and mortality rates after coil occlusion. The overall morbidity and mortality rates associated with both treatments did not vary significantly. That is, in patients with no history of SAH from another lesion the rates were 13.7 and $9.3 \%$ for the surgically and endovascularly treated groups, respectively, whereas in patients with a history of SAH from another lesion the rates were 11 and $7.1 \%$, respectively.

\section{Limitations of the ISUIA and Management Decisions in Patients With Unruptured Aneurysms}

Possible drawbacks of the ISUIA include the potential bias in the enrollment of patients (most of the institutions are busy neurovascular centers and the number of patients with lesions who were enrolled was much smaller than the number of unruptured aneurysms that should be seen in each center each year), the unexplained fact that although the greatest risk of rupture is in lesions larger than $7 \mathrm{~mm}$ in diameter, the great majority of ruptured aneurysms seen were less than $7 \mathrm{~mm}$, and the difference between the results in the retrospective and prospective segments of the study. $9,18,20,23-25$

We believe that the ISUIA is the best evidence available and should be used as a basis for the management of unruptured lesions. That report details the only large, prospective, multicenter study on unruptured intracranial aneurysms that has been published. Most of the series re- 
porting different numbers came from single institutions that are well known for special interest in cerebrovascular disease. The caseload of these centers is certainly affected by referral bias, and the results derived from these series regarding morbidity and mortality rates associated with treatments are not expected to match exactly the results from smaller centers where such expertise is not available. Besides, the bleeding rates in the second part of the ISUIA are quite similar to the rates reported in some of these earlier reports.

The number of cases treated annually also has been demonstrated to influence outcome in intracranial aneurysm treatment, ${ }^{2,8}$ either for craniotomy or for endovascular therapy. Considering that the treatment of unruptured intracranial aneurysms is an elective procedure, and that the benefit of it is extremely dependent on low complication rates, an argument could be made that centers at which a small number of intracranial aneurysms are treated annually, and which therefore have less expertise and higher complication rates, should refer these patients to higher-volume centers.

Because the treatment of unruptured aneurysms is prophylactic, the decision to proceed should be made very carefully. The treatment method must have a very low complication rate. Choosing to treat or not depends on a balance between the risks of the proposed treatment and the natural history of the lesion. The risks associated with treatment will rely on an honest audit at each institution, and they will vary widely depending on surgical and/or endovascular expertise and caseload. The importance of the natural history is completely related to the bleeding rate (Table 2), which is the most feared complication of an unruptured aneurysm. Over the last 20 years the reported bleeding rates have changed from a range of 1.4 to $2.3 \%$ per year ${ }^{10,25}$ to the very low rate of 0.05 to $1 \% \%^{7}$ and in the second part of the ISUIA ${ }^{6}$ again rise to ranges of 0 to $10 \%$ per year.

Using a mathematical predictive model, Leblanc and Worsley ${ }^{13}$ proposed that the treatment of an unruptured intracranial aneurysm would achieve the benefit of adding 1 year free of neurological sequelae to the patient's life, but only if the life expectancy were 19.5 years or more. They assumed a rupture rate of $2 \%$ per year and a surgical risk of $6.5 \%$. Using these numbers and life expectancy tables, they determined that treatment would only be beneficial for men younger than 63.5 years of age and for women who were 68 years old at the time of treatment. Applying the same predictive model to consider the three important factors in the decision process (the treatment risk, bleeding rate, and patient age), neurosurgeons should reevaluate how they determine the merit of prophylactic treatment. If the rupture rate is much lower than $2 \%$ per year, as demonstrated for the majority of aneurysms in the ISUIA, and morbidity and mortality rates at one's institution are greater than $6.5 \%$, then the neurosurgeon should decrease the cutoff age for proposing treatment, perhaps to the early 50 s, to be able to benefit these patients.

\section{CASE ILLUSTRATION}

A case illustration may be the best way to demonstrate decision-making behavior. One of our patients, a 65-yearold right-handed man, presented with tinnitus and this re- sulted in a head CT scan being obtained (Fig. 1). Results of the clinical examination were normal. The patient did not have a headache. An incidental right-sided MCA aneurysm was identified using noninvasive imaging (Fig. 2). The patient had no significant medical history aside from well-controlled hypertension and a history of smoking. He looked very well for his age. The multidisciplinary group proposed treatment and the unfavorable neck anatomy resulted in a recommendation for surgical clip occlusion. The patient underwent successful and uncomplicated craniotomy, with a postoperative angiogram demonstrating obliteration of the lesion (Fig. 3). His 5-year risk was estimated to be $2.6 \%$ ( $\sim 0.5 \%$ per year), much less than the $2 \%$ per year used in the model by Leblanc and Worsley. ${ }^{13}$ Many neurosurgeons reviewing this case would likely have done the same thing, but with new evidence indicating a much lower annual risk of bleeding, maybe our group has not added significantly to his event-free survival. The purpose of presenting this case is to illustrate the care that must be taken with such treatment decisions.

\section{CONCLUSIONS}

The management of unruptured intracranial aneurysms is still controversial. In the last few years, various publications have shown that the risks associated with surgical and endovascular treatments are higher than previously reported in single-institution series. The first part of the ISUIA showed very low rupture rates in individuals with unruptured aneurysms, indicating that no treatment (observation) was an option for many of these lesions. The second part of the ISUIA is the only large, prospective, multicenter trial in the literature on this subject, and in it the findings support predictions of annual hemorrhage rates that are much lower than previously accepted, with

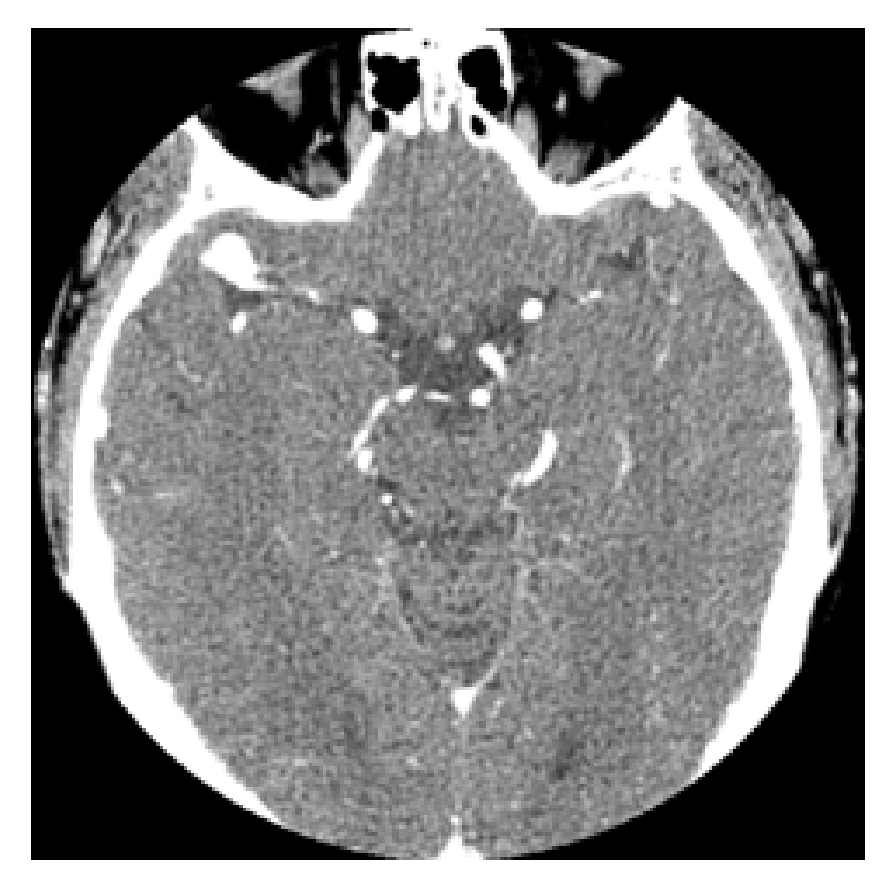

Fig. 1. Enhanced CT scan demonstrating a right-sided MCA aneurysm in a 65-year-old man. 


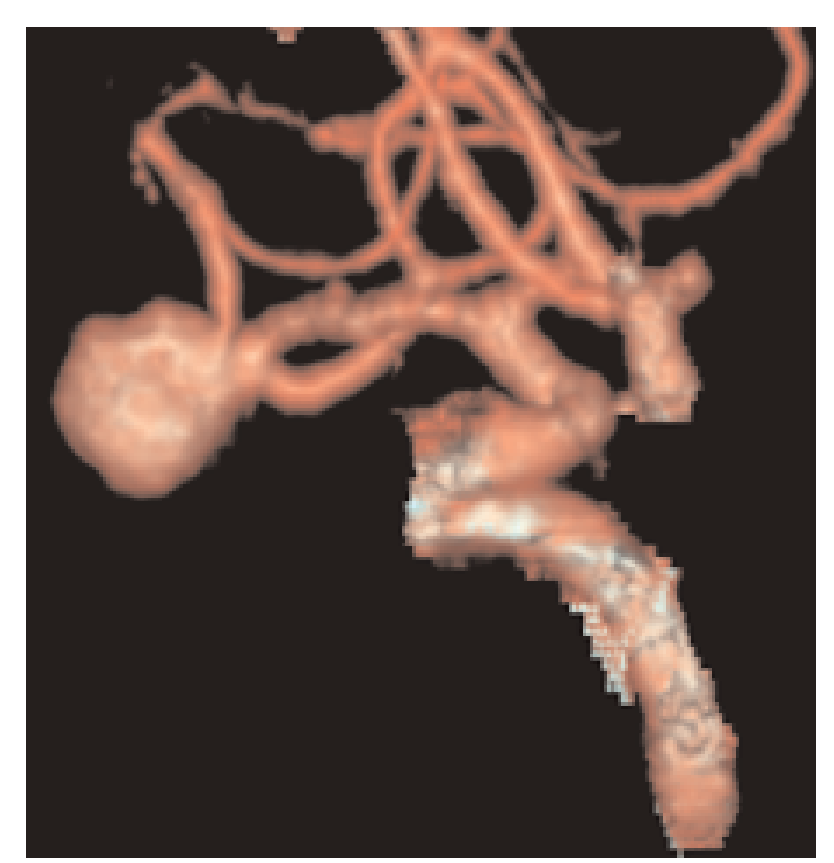

Fig. 2. Three-dimensional reconstruction of a CT angiogram demonstrating a wide-necked, right-sided MCA aneurysm.

treatment-related morbidity and mortality rates higher than often quoted. This will force us to reconsider previous guidelines in light of new information, leading to the following conclusions.

1) Asymptomatic intracavernous aneurysms should not be treated. In large, symptomatic ones the treatment should be individualized.

2) All symptomatic intradural aneurysms should be treated.

3) Incidental aneurysms with diameters less than $7 \mathrm{~mm}$ should not be treated. Nevertheless, lesions approaching $7 \mathrm{~mm}$ occurring in younger patients should be considered

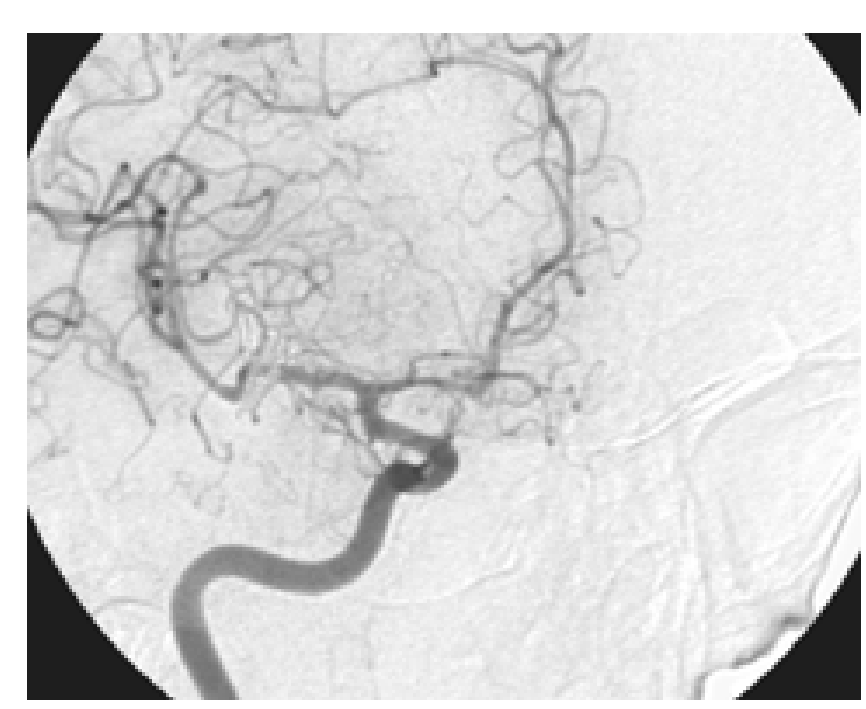

Fig. 3. Postoperative angiogram of the right carotid artery demonstrating obliteration of the MCA aneurysm. for treatment. The cutoff age for treatment consideration will likely drop a decade from the one proposed in previous guidelines, given new, lower risks predicted for the natural history of hemorrhage.

4) Aneurysms found in association with a ruptured lesion and those with diameters larger than $7 \mathrm{~mm}$ deserve strong consideration for treatment, especially in young patients.

5) Aneurysms located at the PCoA and those in the posterior circulation, especially the basilar tip, have higher rupture rates, and therefore deserve special consideration for treatment.

6) Endovascular treatment should be considered as a treatment option for incidental aneurysms.

The care of a patient with an unruptured intracranial aneurysm remains challenging. The continued study of these lesions around the world will lead to new information and perhaps the continuation of a "moving target" designation with respect to their hemorrhagic behavior. Surgeons treating patients who present with this disease should continually adjust to this new information and audit their own results to do the best for their patients.

\section{References}

1. Bederson JB, Awad IA, Wiebers DO, et al: Recommendations for the management of patients with unruptured intracranial aneurysms: A Statement for healthcare professionals from the Stroke Council of the American Heart Association. Stroke 31: 2742-2750, 2000

2. Berman MF, Solomon RA, Mayer SA, et al: Impact of hospitalrelated factors on outcome after treatment of cerebral aneurysms. Stroke 34:2200-2207, 2003

3. Brennan JW, Schwartz ML: Unruptured intracranial aneurysms: appraisal of the literature and suggested recommendations for surgery, using evidence-based medicine criteria. Neurosurgery 47:1359-1372, 2000

4. Britz GW, Salem L, Newell DW, et al: Impact of surgical clipping on survival in unruptured and ruptured cerebral aneurysms: a population-based study. Stroke 35:1399-1403, 2004

5. Cook DJ, Guyatt GH, Laupacis A, et al: Rules of evidence and clinical recommendations on the use of antithrombotic agents.Chest 102 (Suppl 4):305S-311S, 1992

6. The International Study of Unruptured Intracranial Aneurysms Investigators: Unruptured intracranial aneurysms: natural history, clinical outcome, and risks of surgical and endovascular treatment. Lancet 362:103-110, 2003

7. The International Study of Unruptured Intracranial Aneurysms Investigators: Unruptured intracranial aneurysms-risk of rupture and risks of surgical intervention. $\mathbf{N}$ Engl J Med 339: $1725-1733,1998$

8. Johnston SC, Zhao S, Dudley RA, et al: Treatment of unruptured cerebral aneurysms in California. Stroke 32:597-605, 2001

9. Juvela S: Recommendations for the management of patients with unruptured intracranial aneurysms. Stroke 32:815-816, 2001

10. Juvela S, Porras M, Heiskanen O: Natural history of unruptured intracranial aneurysms: a long-term follow-up study. J Neurosurg 79:174-182, 1993

11. Juvela S, Porras M, Poussa K: Natural history of unruptured intracranial aneurysms: probability of and risk factors for aneurysm rupture. J Neurosurg 93:379-387, 2000

12. King JT Jr, Berlin JA, Flamm ES: Morbidity and mortality from elective surgery for asymptomatic, unruptured, intracranial aneurysms: a meta-analysis. J Neurosurg 81:837-842, 1994

13. Leblanc R, Worsley KJ: Surgery of unruptured, asymptomatic 
aneurysms: a decision analysis. Can J Neurol Sci 22:30-35, 1995

14. Ogilvy CS, Carter BS: Stratification of outcome for surgically treated unruptured intracranial aneurysms. Neurosurgery 52: 82-88, 2003

15. Orz YI, Hongo K, Tanaka Y, et al: Risks of surgery for patients with unruptured intracranial aneurysms. Surg Neurol 53: 21-29, 2000

16. Raaymakers TW: Functional outcome and quality of life after angiography and operation for unruptured intracranial aneurysms. On behalf of the MARS Study Group. J Neurol Neurosurg Psychiatry 68:571-576, 2000

17. Raaymakers TWM, Rinkel GJE, Limburg M, et al: Mortality and morbidity of surgery for unruptured intracranial aneurysms: a meta-analysis. Stroke 29:1531-1538, 1998

18. Raftopoulos C, Goffette P, Vaz G, et al: Surgical clipping may lead to better results than coil embolization: results from a series of 101 consecutive unruptured intracranial aneurysms. Neurosurgery 52:1280-1290, 2003

19. Towgood K, Ogden JA, Mee E: Neurological, neuropsychological, and psychosocial outcome following treatment of unruptured intracranial aneurysms: a review and commentary. J Int Neuropsychol Soc 10:114-134, 2004

20. Tsutsumi K, Ueki K, Morita A, et al: Risk of rupture from incidental cerebral aneurysms. J Neurosurg 93:550-553, 2000
21. Wiebers DO, Whisnant JP, O'Fallon WM: The natural history of unruptured intracranial aneurysms. N Engl J Med 304: 696-698, 1981

22. Wiebers DO, Whisnant JP, Sundt TM Jr, et al: The significance of unruptured intracranial saccular aneurysms. J Neurosurg 66:23-29, 1987

23. Winn HR: Section Overview: unruptured aneurysms. J Neurosurg 96:1-2, 2002

24. Winn HR, Jane JA Sr, Taylor J, et al: Prevalence of asymptomatic incidental aneurysms: review of 4568 arteriograms. J Neurosurg 96:43-49, 2002

25. Yasui N, Suzuki A, Nishimura H, et al: Long-term follow-up study of unruptured intracranial aneurysms. Neurosurgery 40: 1155-1160, 1997

Manuscript received September 20, 2004.

Accepted in final form October 5, 2004.

Address reprint requests to: M. C. Wallace, MD, MSc, 399 Bathurst Street 4-450, Toronto Western Hospital, Toronto, Ontario, Canada, M5T 2S8. email: chris.wallace@uhn.on.ca. 